\title{
Improving Image Quality in Electrical Impedance Tomography (EIT) Using Projection Error Propagation-Based Regularization (PEPR) Technique: A Simulation Study
}

\author{
Tushar Kanti Bera ${ }^{1}$, Samir Kumar Biswas ${ }^{2}$, K. Rajan ${ }^{2}$ and J. Nagaraju ${ }^{1}$ \\ 1. Department of Instrumentation and Applied Physics, Indian Institute of Science, Bangalore - 560012, India \\ 2. Department of Physics, Indian Institute of Science, Bangalore - 560012, India \\ 3. E-mail any correspondence to: solarjnr@isu.iisc.ernet.in
}

\begin{abstract}
A Projection Error Propagation-based Regularization (PEPR) method is proposed and the reconstructed image quality is improved in Electrical Impedance Tomography (EIT). A projection error is produced due to the misfit of the calculated and measured data in the reconstruction process. The variation of the projection error is integrated with response matrix in each iteration and the reconstruction is carried out in EIDORS. The PEPR method is studied with the simulated boundary data for different inhomogeneity geometries. Simulated results demonstrate that the PEPR technique improves image reconstruction precision in EIDORS and hence it can be successfully implemented to increase the reconstruction accuracy in EIT.
\end{abstract}

Keywords: electrical impedance tomography, projection error propagation-based regularization (PEPR), simulated boundary potentials, image reconstruction, forward problem, inverse problem, finite element method.

\section{Introduction}

Electrical Impedance Tomography (EIT) [1] is being researched in different areas of science and technology due to its many advantages [2-4] over other computed tomographic techniques [5]. Being a non-invasive, nonradiating, non-ionizing and inexpensive methodology, EIT has been extensively studied in clinical diagnosis [6], biomedical engineering [7] and biotechnology [8]. Attempts are also being made to develop a better medical-EIT system (Fig.-1) for different clinical investigations [9-18] and long time patient monitoring. The first impedance imaging system, the Impedance Camera, was constructed by Henderson and Webster to study pulmonary edema in 1978 [19]. EIT has, generally, poor signal to noise ratio [7], poor spatial resolution [20] and it is highly sensitive to modeling parameters [21] such as the electrodes and phantom geometry experimental errors in the boundary data. As a result EIT is not yet accepted as the gold method in medical imaging technology. Therefore improving the image quality and spatial resolution is a big challenge in the field of impedance imaging. Using noninvasive boundary measurements EIT reconstructs the images of impedance distribution (conductivity or resistivity or permittivity) of the closed domain under test.

Conductivity reconstruction in EIT is a nonlinear, highly ill-posed [21] inverse problem in which a small amount of noise in the boundary data can lead to enormous errors in the estimates. In fact, like many other inverse problems encountered in physics, EIT is a highly ill-posed non-linear inverse problem which causes the instability of the solution due to errors on the observed data. Being an illposed problem EIT needs a regularization technique [21] to constrain its solution space. Regularization technique is implemented to convert the ill-posed problem into a well posed problem using a suitable regularization parameter $(\lambda)$. Regularization in inverse problems not only decreases the ill-posed characteristics of the inverse matrix but also, it improves the reconstructed image quality [22]. The standard Tikhonov regularization [23] is the simplest method to implement, in which the regularization matrix is proportional to identity. Since the physical attenuation phenomena responsible for the illposed nature of the EIT problem is not taken into account, the standard Tikhonov regularization cannot provide a satisfactory solution in image reconstruction for EIT [24].

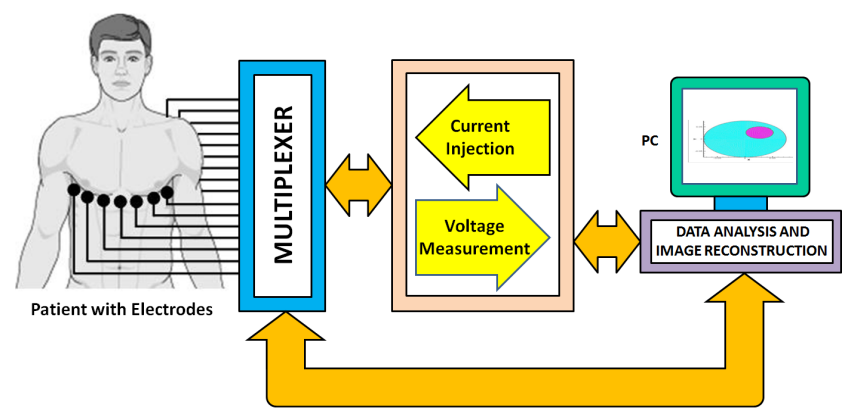

Fig.1: An EIT system with electrode array on patient under test.

In this context a Projection Error Propagation-based Regularization (PEPR) method [25] is proposed to improve the reconstructed image quality in static EIT, which produces an image of the absolute resistivity distribution of the medium. The PEPR method is studied with resistivity reconstruction in EIT using simulated data. In the PEPR method the regularization parameter is set as a function of 
the projection error which is produced by the mismatch between the calculated and measured data. In the first iteration, the regularization parameter is calculated with the projection error developed by the boundary data estimated by the forward solver for an initial guessed resistivity. According to the resistivity update vector calculated in all the other iterations, regularization parameter is also modified. At each iteration, projection error varies according to the misfit between the model predicted data and experimental data. The variation of mismatch data is integrated with the response matrix and the reconstruction is conducted.

To illustrate the performance of this method, resistivity images are reconstructed using EIDORS (Electrical Impedance Tomography and Diffuse Optical Tomography Reconstruction Software) [26-27] with PEPR and compared with the images reconstructed with Levenberg-Marquardt Regularization (LMR) [25]. To study the PEPR method, reconstructed image parameters, normalized projection error (error due to the voltage mismatch) and the normalized solution error norm are studied for different iterations. Result show that the PEPR technique improves image reconstruction precision in EIDORS. It is also observed that the PEPR technique can improve image quality more effectively and reduces the background noise.

\section{Materials and methods}

\section{EIT}

Electrical Impedance Tomography is a non linear inverse problem [21] in which the electrical conductivity distribution of a closed domain $(\mathbf{\Omega})$ in an object under test called phantom, [28-29] is reconstructed from the surface potential at the boundary ( $\partial \mathbf{\Omega})$ developed by injecting a sinusoidal current signal [30]. A low frequency $(10 \mathrm{kHz}-$ $1 \mathrm{MHz}$ ) and low magnitude constant current is injected through an array of 16 surface electrodes [31] surrounding the domain to be imaged using different electrode switching protocols [32] and the boundary potentials are measured [33] although, the boundary currents can also be measured for an applied voltage signal in Applied Potential Tomography [34]. The voltage data collected by the data acquisition system is then processed by an image reconstruction algorithm in a $\mathrm{PC}$.

\section{Mathematical Model}

To calculate the nodal potential for a known conductivity a relationship can be established between the electrical conductivity $(\boldsymbol{\sigma})$ and spatial potential $(\boldsymbol{\Phi})$. Electro-dynamics of EIT is governed by a nonlinear partial differential equation, called the Governing Equation [2-4] of Electrical Impedance Tomography, which is given by,

$$
\nabla \bullet \sigma \nabla \Phi=0
$$

\section{Forward and Inverse Problem}

A relation can be obtained between the voltage measurements made on the boundary $(\partial \boldsymbol{\Omega})$ and the domain conductivity can be found [35-36] as,

$$
\Phi=[\mathrm{K}(\sigma)][\mathrm{I}]
$$

Where $\boldsymbol{\sigma}$ is elemental conductivity values, $\boldsymbol{\Phi}$ is the vector of nodal potential and $\mathbf{K}$ is the transformation matrix constructed from the elemental conductivities and nodal coordinates.

If $\mathbf{K}$ and $\mathbf{I}$ are known, Eq.-2 can be solved numerically using the finite element method (FEM) [37] to calculate the nodal potentials of the domain for the known conductivity $(\boldsymbol{\sigma})$. It is known as the "forward problem". Using the GaussNewton method [38] applied on EIT, the update vector of $\sigma$ [35-36] can be expressed as:

$$
\sigma=[\mathrm{Q}]\left[\Phi_{\mathrm{d}}\right]
$$

Where, $\mathbf{Q}$ is a function of the Jacobian matrix (J) [35-36] and regularization parameters [35-36]. $\boldsymbol{\Phi}_{\mathbf{d}}$ is the mismatch vector between calculated boundary potential $\left(\mathbf{V}_{\mathbf{c}}\right)$ and measured boundary potential $\left(\mathbf{V}_{\mathbf{m}}\right)$. That means if the matrix $\mathbf{Q}$ and the surface potentials $\boldsymbol{\Phi}$ are known then the elemental conductivity $\left(\boldsymbol{\sigma}_{\mathbf{e}}\right)$ can be mapped. This is known as the "inverse problem" which is discussed in the next section. Using the Modified Newton Raphson (MNR) iterative technique [35-36], a suitably assumed conductivity vector (initial guess), $\left[\boldsymbol{\sigma}_{\mathbf{0}}\right]$, is modified to $\left[\boldsymbol{\sigma}_{\mathbf{0}}+\Delta \boldsymbol{\sigma}\right]$ for achieving a specified error limit in the calculated and measured voltage $([\Delta \sigma]$ denotes the conductivity update).

\section{Image Reconstruction: Gauss-Newton Approach}

Electrical conductivity imaging is a highly nonlinear and ill-posed inverse problem. The response matrix $\left[\mathbf{J}^{\mathbf{T}} \mathbf{J}\right]$ is a singular matrix. Hence in EIT, a minimization algorithm [35-36, 38-39] is used to obtain the approximate solution of the ill-posed inverse problem. In the minimization algorithm, the objective function formed by the difference between the experimental measurement data $\left(\mathbf{V}_{\mathbf{m}}\right)$ and the computationally predicted data $\left(\mathbf{V}_{\mathbf{c}}\right)$ is minimized. Generally in inverse problems a least square solution [3536] of a minimized object function (s) [38-39] obtained from the calculated voltage data and the measured voltage data is searched by a Gauss-Newton method based numerical approximation algorithm (explained in the next sub-section) called the inverse solver [40].

If $\mathbf{V}_{\mathbf{m}}$ is the measured voltage matrix and $\mathbf{f}$ is a function mapping an E-dimensional ( $\mathrm{E}$ is the number of element in the FEM mesh) impedance distribution into a set of $M$ (number of the measured data available) approximate measured voltage, then the Gauss-Newton algorithm 
[35-36, 38-39] tries to find a least square solution of the minimized object function s defined as [38-43]:

$$
\mathrm{s}_{\mathrm{r}}=\frac{1}{2}\left\|\mathrm{~V}_{\mathrm{m}}-\mathrm{f}\right\|^{2}+\frac{1}{2} \lambda\|\mathrm{G} \sigma\|^{2}
$$

Where, $\mathbf{s}_{\mathbf{r}}$ is the constrained least-square error of the reconstructions, $\mathbf{G}$ is the regularization operator and $\lambda$ is a positive scalar and called as the regularization coefficient.

$$
\mathrm{s}_{\mathrm{r}}=\frac{1}{2}\left(\mathrm{~V}_{\mathrm{m}}-\mathrm{f}\right)^{\mathrm{T}}\left(\mathrm{V}_{\mathrm{m}}-\mathrm{f}\right)+\frac{1}{2} \lambda(\mathrm{G} \sigma)^{\mathrm{T}}(\mathrm{G} \sigma)
$$

Now, differentiating Eq.-5 w.r.t. $\boldsymbol{\sigma}$, it reduces to:

$$
\mathrm{s}_{\mathrm{r}}{ }^{\prime}=-\left(\mathrm{f}^{\prime}\right)^{\mathrm{T}}\left(\mathrm{V}_{\mathrm{m}}-\mathrm{f}\right)+\lambda(\mathrm{G})^{\mathrm{T}}(\mathrm{G} \sigma)
$$

Where the term $\mathrm{f}^{\prime}=\mathbf{J}$ is known as the Jacobian matrix of size $\mathrm{g} \times \mathrm{h}$ and which is defined by $[36,39]$ :

$$
\mathrm{J}=\left[\mathrm{f}^{\prime}\right]_{\mathrm{gh}}=\frac{\partial \mathrm{f}_{\mathrm{g}}}{\partial \sigma_{\mathrm{h}}}
$$

Where,

$\mathrm{e}=1,2 \ldots \mathrm{E}[\mathrm{E}=$ number of elements in the FEM mesh],

$\mathrm{h}=1,2 \ldots \mathrm{M}[\mathrm{M}=$ (number of data measured per current

projections $(d)) \times($ number of current projections $(p))]$

By the inherent ill-posed nature of EIT, the $\left[\mathrm{f}^{\prime}\right]^{\mathrm{T}}$ matrix in Eq.-6 is always ill-conditioned [22], and hence small measurement errors will make the solution of Eq. -6 change greatly which is made well posed by the regularization term incorporated. Differentiating Eq.-6 w.r.t. $\boldsymbol{\sigma}$, it reduces to:

$$
\mathrm{s}_{\mathrm{r}}{ }^{\prime \prime}=(\mathrm{J})^{\mathrm{T}}(\mathrm{J})-\left(\mathrm{f}^{\prime \prime}\right)^{\mathrm{T}}\left(\mathrm{V}_{\mathrm{m}}-\mathrm{f}\right)+\lambda \mathrm{G}^{\mathrm{T}} \mathrm{G}
$$

By the Gauss Newton (GN) method,

$$
\Delta \sigma=-\frac{\mathrm{s}_{\mathrm{r}}^{\prime}}{\mathrm{s}_{\mathrm{r}}^{\prime \prime}}=\frac{(\mathrm{J})^{\mathrm{T}}\left(\mathrm{V}_{\mathrm{m}}-\mathrm{f}\right)-\lambda(\mathrm{G})^{\mathrm{T}}(\mathrm{G} \sigma)}{(\mathrm{J})^{\mathrm{T}}(\mathrm{J})-\left(\mathrm{f}^{\prime \prime}\right)^{\mathrm{T}}\left(\mathrm{V}_{\mathrm{m}}-\mathrm{f}\right)+\lambda \mathrm{G}^{\mathrm{T}} \mathrm{G}}
$$

Neglecting higher terms, the update vector reduces to:

$$
\Delta \sigma=\frac{(\mathrm{J})^{\mathrm{T}}\left(\mathrm{V}_{\mathrm{m}}-\mathrm{f}\right)-\lambda(\mathrm{G})^{\mathrm{T}}(\mathrm{G} \sigma)}{(\mathrm{J})^{\mathrm{T}}(\mathrm{J})+\lambda \mathrm{G}^{\mathrm{T}} \mathrm{G}}
$$

Replacing $\mathbf{G}^{\mathbf{T}} \mathbf{G}$ by $\mathbf{I}$ (Identity matrix) Eq.-10 reduces to:

$$
\Delta \sigma=\frac{\mathrm{J}^{\mathrm{T}}\left(\mathrm{V}_{\mathrm{m}}-\mathrm{f}\right)-\lambda \mathrm{I} \sigma}{\mathrm{J}^{\mathrm{T}} \mathrm{J}+\lambda \mathrm{I}}
$$

In general, for $\mathrm{k}^{\text {th }}$ iteration ( $\mathrm{k}$ is a positive integer), the conductivity update vector of Eq.-11 is reduced to:

$$
\Delta \sigma_{\mathrm{k}}=\left[\left[\mathrm{J}_{\mathrm{k}}\right]^{\mathrm{T}}\left[\mathrm{J}_{\mathrm{k}}\right]+\lambda \mathrm{I}\right]^{-1}\left[\left[\mathrm{~J}_{\mathrm{k}}\right]^{\mathrm{T}}(\Delta \mathrm{V})_{\mathrm{k}}-\lambda \mathrm{I} \sigma_{\mathrm{k}}\right]
$$

Where $\mathbf{J}_{\mathbf{k}}$ and $(\Delta \mathrm{V})_{\mathrm{k}}$ are the Jacobian and voltage difference matrix respectively at the $\mathrm{k}^{\text {th }}$ iteration.

Thus the Gauss-Newton method based inverse solver algorithm gives a regularized solution of the conductivity distribution for the $\mathrm{k}^{\text {th }}$ iteration as:

$\sigma_{\mathrm{k}+1}=\sigma_{\mathrm{k}}+\left[\left[\mathrm{J}_{\mathrm{k}}\right]^{\mathrm{T}}\left[\mathrm{J}_{\mathrm{k}}\right]+\lambda \mathrm{I}\right]^{-1}\left[\left[\mathrm{~J}_{\mathrm{k}}\right]^{\mathrm{T}}(\Delta \mathrm{V})_{\mathrm{k}}-\lambda \mathrm{I} \sigma_{\mathrm{k}}\right]$

\section{PEPR Method}

In this paper a PEPR method is proposed to improve the reconstructed image quality in EIT and the resistivity reconstruction is studied in EIDORS. Generally, the choice of an appropriate regularization can be evaluated or found empirically [25] and the regularization parameter is related to an objective function [38-39]. In our study, the projection error is utilized as the objective function; hence the regularization parameter would be expected to be related to the projection error. Furthermore, the value of regularization is required to vary with projection error. That is to say, a greater regularization value is needed for a larger projection error during iterations. If the projection error is very low, only a small regularization value is needed to regulate the ill-posed process [25]. Based on these considerations, we define the adaptive regularization parameter $\boldsymbol{\lambda}$ as follows:

$$
\lambda=\frac{\Psi}{2+\mathrm{e}^{-\Delta \Phi}}
$$

Where, $\Psi$ is a const (taken as 0.01 ), $\Delta \Phi$ is the projection error which is defined as the L-2 norm of the difference between the calculated data and the measured data. Hence the regularization parameter $\lambda$ in the PEPR method is given by:

$$
\lambda=\frac{0.01}{2+\mathrm{e}^{-\left\|\mathrm{V}_{\mathrm{c}}-\mathrm{V}_{\mathrm{m}}\right\|^{2}}}
$$

In formula (15), the regularization parameter $\lambda$ varies in the range from $0.01 / 3$ to $0.01 / 2$. It includes two primary considerations: (1) Due to the ill-posed characteristic of the inverse problem, the regularization should not be reduced with iterations to a too small value. (2) The regularization value should be lower than the maximum value of diagonal elements in the matrix $\mathbf{J J}^{\mathbf{T}}$ (calculated by the MATLAB 
code: $\left.\max \left(\max \left(\mathbf{J} \mathbf{J}^{\mathbf{T}}\right)\right)\right)$. In the PEPR method the regularization parameter is set as a function of the projection error which is produced by the mismatch between the calculated and measured data. In the first iteration, the regularization parameter is calculated with the projection error developed by the boundary data estimated by the forward solver for an initial guessed resistivity. According to the resistivity update vector calculated, the regularization parameter is also modified in all the other iterations. Hence at each iteration, the projection error varies according to the misfit between the model predicted data and experimental data. The variation of mismatch data is integrated with the response matrix and the reconstruction is carried out.

The $\mathbf{J}^{\mathbf{T}} \mathbf{J}$ matrix is formed by the first derivative ( $\left.\mathbf{J}\right)$ of the forward solution (FS) and $\mathbf{I}$ is the approximation of the Hessian [39] which is, actually, the second derivative of the FS. Generally, the order of magnitude of Hessian is less than the $\mathbf{J}^{\mathbf{T}} \mathbf{J}$ due to the higher order derivative.

Hence, in Eq.-13, I acts as the quadratic term which is formed with the maximum value of $\mathbf{J}^{\mathbf{T}} \mathbf{J}$. The magnitude of the diagonal elements of $\lambda \mathbf{I}$ should be less than the maximum value of $\mathbf{J}^{\mathbf{T}} \mathbf{J}$. The identity matrix $\mathbf{I}$ is formed with the diagonal values equal to the maximum value of the matrix $\mathbf{J}^{\mathbf{T}} \mathbf{J}$. In the LMR method the $\lambda$ is taken as $10^{-1}$ in the first iteration and it is then modified as $\lambda / \mathrm{k}$ in the modified EIDORS, where $\mathrm{k}$ is the number of iterations. For further analysis the resistivity reconstruction is also conducted with a range of regularization parameters.

It is observed that the projection error in LMR and PEPR becomes minimum at $\lambda=0.1$ and $\Psi=0.01$ respectively. That is why in the first iteration in LMR $\lambda$ is taken as 0.1 and in PEPR $\lambda$ is calculated with $\Psi=0.01$. Furthermore, for better understanding the regularization effects of both the methods, $\lambda$ is calculated in PEPR technique (using the Eq.-14) with $\Psi=0.01$. The result is compared with the reconstruction obtained in LMR in which the iteration starts with $\lambda=0.01$ and then it is decreased by a factor of $\sqrt{10}$ for the other iterations [25].

\section{Resistivity Reconstruction and Analysis}

Resistivity images are reconstructed using simulated boundary data in EIDORS with the PEPR and LMR method and the results are compared. Resistivity images are reconstructed with simulated data for different inhomogeneity geometries. Circular objects (diameter $=60$ $\mathrm{mm}$, resistivity $=33 \Omega \mathrm{m}$ ) with a homogeneous background medium $($ diameter $(\mathrm{D})=150 \mathrm{~mm}$, resistivity $=2.5 \Omega \mathrm{m})$ are simulated for boundary data generation. Resistivity images are also reconstructed from the boundary data combined with random noise of different percentages. Noisy data are used for reconstruction with the PEPR and LMR methods and the images are compared.
To analyze the proposed method, the normalized projection error (error due to the voltage mismatch), $\mathrm{E}_{\mathrm{V}}$, is calculated in each iteration as:

$$
\mathrm{E}_{\mathrm{V}}=\frac{1}{2}\left\|\mathrm{~V}_{\mathrm{m}}-\mathrm{V}_{\mathrm{c}}\right\|^{2}
$$

The normalized solution error norm $\left(E_{\rho}\right)$ is also calculated in each iteration as:

$$
\mathrm{E}_{\rho}=\frac{\left\|\rho_{\text {reconstructed }}-\rho_{\text {true }}\right\|}{\left\|\rho_{\text {true }}\right\|}
$$

A contrast to noise ratio (CNR) [44-45] is calculated for the reconstructed images in this work to evaluate the reconstructed images with different regularization techniques. CNR is defined as the ratio of the difference between the average inhomogeneity resistivity $\left(\mathrm{IR}_{\mathrm{Mean}}\right)$ and the average background resistivity $\left(\mathrm{BR}_{\mathrm{Mean}}\right)$ divided by the weighted average of the standard deviations in the IR $\left(\mathrm{SD}_{\mathrm{IR}}\right)$ and $\mathrm{BR}\left(\mathrm{SD}_{\mathrm{BR}}\right)$ :

$$
\mathrm{CNR}=\frac{\mathrm{IR}_{\text {Mean }}-\mathrm{BR}_{\text {Mean }}}{\left(\omega_{\mathrm{I}}\left(\mathrm{SD}_{\mathrm{IR}}\right)^{2}+\omega_{\mathrm{B}}\left(\mathrm{SD}_{\mathrm{BR}}\right)^{2}\right)^{\frac{1}{2}}}
$$

Where $\omega_{\mathrm{I}}$ is the fraction of the area of the region of interest with respect to the area of the whole image; $\omega_{\mathrm{B}}$ is defined as $\omega_{B}=1-\omega_{I}$. $I_{\text {Mean }}$ and $B R_{\text {Mean }}$ are the mean values of the inhomogeneity and the background regions in the reconstructed images.

Percentage of contrast recovery (PCR) [46] is calculated for the images reconstructed by all the regularization technique to compare the reconstruction accuracy. PCR in EIT is defined as the difference between the averaged resistivity within the reconstructed image $\left(\mathrm{IR}_{\mathrm{Mean}}\right)$ and the reconstructed background $\left(\mathrm{BR}_{\mathrm{Mean}}\right)$ divided by the difference between the original resistivity of the inhomogeneity ( $\left.\mathrm{IR}_{\text {Original }}\right)$ and the background $\left(\mathrm{BR}_{\text {Original }}\right)$. Hence mathematically the PCR is obtained by the equation:

$$
\mathrm{PCR}=\frac{\mathrm{IR}_{\text {Mean }}-\mathrm{BR}_{\text {Mean }}}{\mathrm{IR}_{\text {Original }}-\mathrm{BR}_{\text {Original }}} \times 100
$$

Coefficient of Contrast (COC) in EIT is defined as the ratio of the mean inhomogeneity resistivity $\left(\mathrm{IR}_{\text {mean }}\right)$ to mean background resistivity $\left(\mathrm{BR}_{\text {mean }}\right)$ though in some literature this ratio is termed as the contrast recovery [47]:

$$
\mathrm{COC}=\frac{\mathrm{IR}_{\text {Mean }}}{\mathrm{BR}_{\text {Mean }}}
$$


CNR, PCR, COC, $\mathrm{IR}_{\text {Mean }}$ and $\mathrm{IR}_{\mathrm{Max}}$ (maximum values of the reconstructed inhomogeneity resistivity) are calculated for all the images and are compared to assess the reconstructed image quality with PEPR. To evaluate the reconstructed images the elemental resistivity along the phantom diameter (D), connecting the centre of the reconstructed object and the centre of the phantom, is plotted against the length of the phantom diameter. This resistivity plot is termed as the diametric resistivity plot (DRP). The normalized projection error (error due to the voltage mismatch) and the normalized solution error norm are studied for different iterations.

\section{Results}

Imaging with noiseless data for different object positions in LMR $($ Lambda $=0.1)$ and PEPR $(P s i=0.01)$

The reconstruction of the simulated phantom with a circular object near electrode 1 (Fig.-2a) shows that the CNR of the resistivity image reconstructed with LMR (Fig.-2b) is 3.08 whereas the CNR of the resistivity image with PEPR (Fig.2c) method (Table-1) is 3.55. For the same simulated phantom, the PCR of the resistivity image with LMR technique is 32.52 whereas the PCR with PEPR is 46.57 (Table-1). It is also noticed that the COC of the resistivity image with LMR technique is 2.05 whereas the COC with PEPR is 2.46 (Table-1). It is observed that the DRP of the resistivity image with PEPR (Fig.-2d) is more similar to the DRP of the original object.

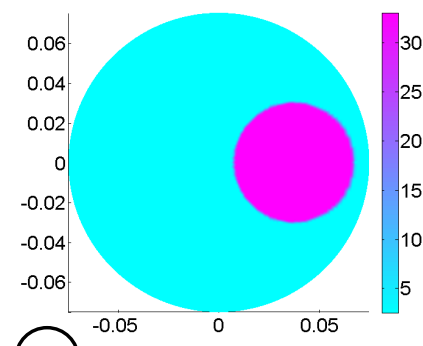

\section{(a)}
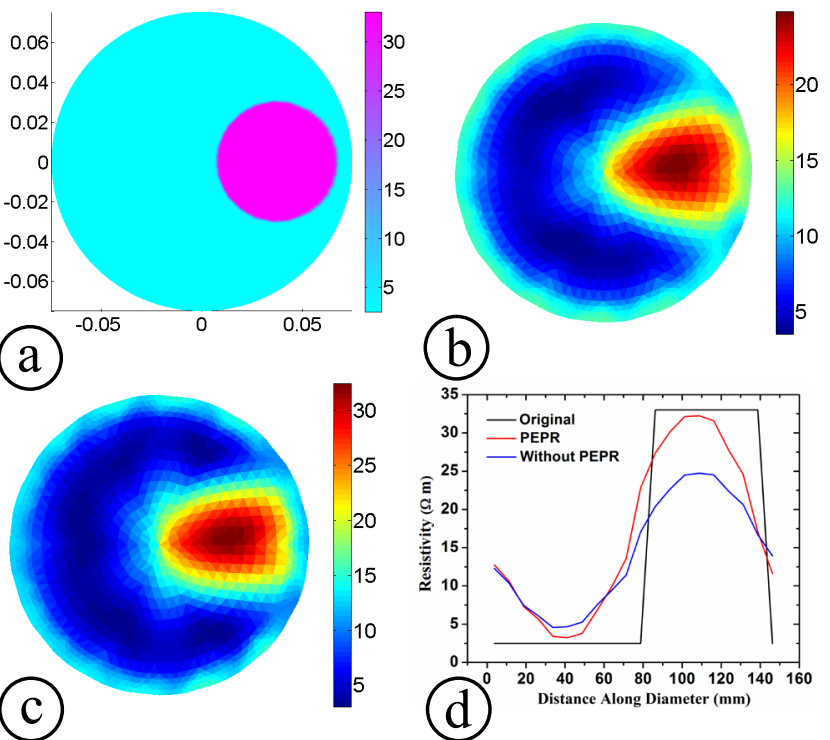

Fig.2: Resistivity imaging for object near electrode 1: (a) Original object, (b) with LMR, (c) with PEPR, (d) DRP of the images.

Table-1: CNR, PCR and COC of reconstructed images of the object near electrode 1

\begin{tabular}{|l|l|l|l|}
\hline Regularization & CNR & PCR & COC \\
\hline LMR & 3.08 & 32.52 & 2.05 \\
\hline PEPR & 3.55 & 46.57 & 2.46 \\
\hline
\end{tabular}

For the object near electrode 3 (Fig.-3a), the reconstructed images (Fig.-3b-3c) show that the CNR of the resistivity image with LMR technique is 2.99. On the other hand, CNR with PEPR is 3.51 (Table-2). The PCR of the resistivity image with LMR technique is 31.33 whereas the PCR with PEPR is 46.26 (Table-2). It is also noticed that the COC of the resistivity image with LMR is 2.01 but with PEPR, it is 2.45 (Table-2). Result show that the DRP of the resistivity image with PEPR (Fig.-3d) is more similar to the DRP of the original object.

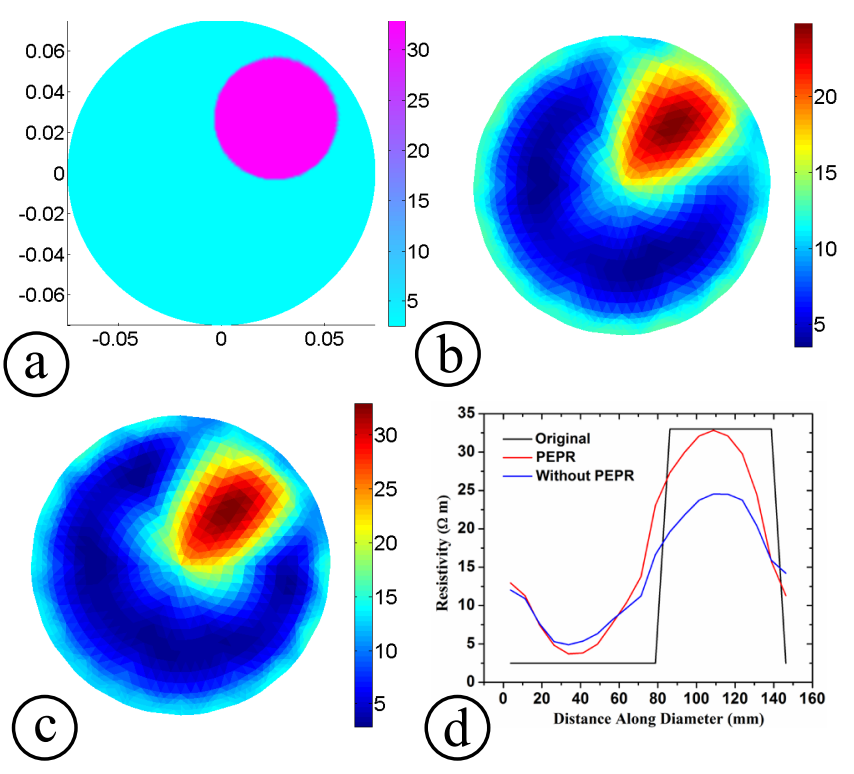

Fig.3: Resistivity imaging for object near electrode 3: (a) Original object, (b) with LMR, (c) with PEPR, (d) DRP of the images.

Table-2: CNR, PCR and COC of reconstructed images of the object near electrode 3

\begin{tabular}{|l|l|l|l|}
\hline Regularization & CNR & PCR & COC \\
\hline LMR & 2.99 & 31.33 & 2.01 \\
\hline PEPR & 3.51 & 46.26 & 2.45 \\
\hline
\end{tabular}

It is observed that for the simulated phantom with a circular object near electrode 5 (Fig.-4a), the CNR of the image reconstructed with LMR (Fig.-4b) is 2.92 whereas the CNR of the resistivity image with PEPR (Fig.-4c) is 3.46 (Table-3). It is also noticed that the PCR of the image with LMR is 30.32 whereas the PCR with PEPR is 44.74. Results also show that the COC with LMR is 1.98 (Table-3) whereas with PEPR technique it is 2.40 . It is observed that the DRP of the resistivity image with PEPR (Fig.-4d) is more similar to the DRP of the original object.

Imaging with noisy data for different object positions in LMR $($ Lambda $=0.1)$ and PEPR $(P s i=0.01)$

Resistivity images are reconstructed from the boundary data with $25 \%$ random noise for the object near electrode 1,3 and 5. Images reconstructed from the boundary data with added noise for the object near electrode 1 show that the 
CNR of the resistivity image with LMR technique (Fig.-5a) is 1.81. On the other hand, CNR of the image with PEPR (Fig.-5b) is 3.17 (Table-4). The PCR of the resistivity image with LMR technique is 27.29 whereas the PCR with PEPR is 63.79 (Table-4). It is also noticed that the COC of the resistivity image with LMR is 1.85 but with PEPR it is 2.90 (Table-4). Result shows that the DRP of the resistivity image with PEPR (Fig.-5c) is more similar to the DRP of the original object.

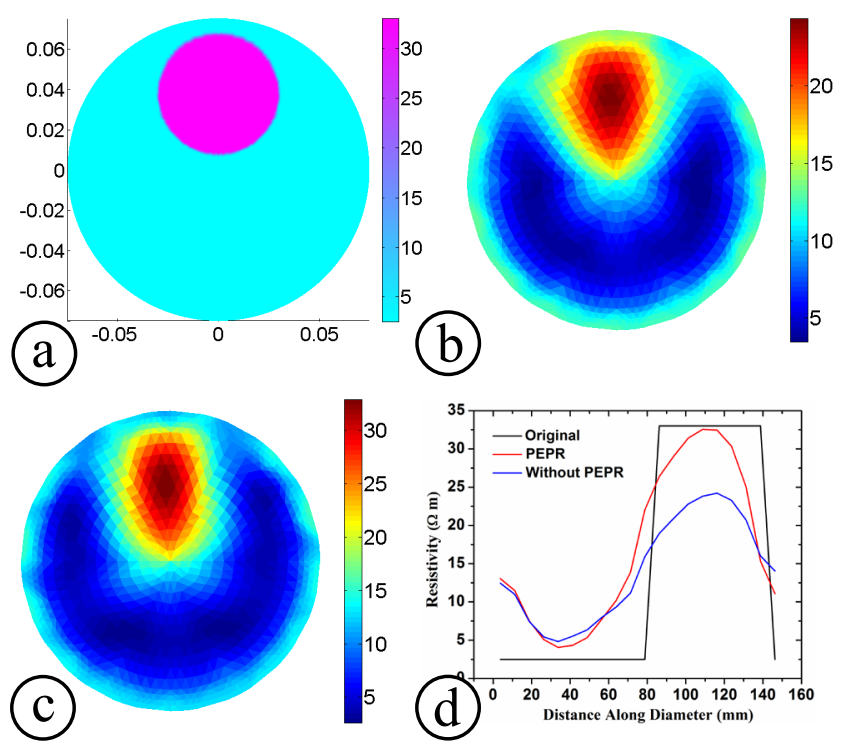

Fig.-4: Resistivity imaging for object near electrode 5: (a) Original object, (b) with LMR, (c) with PEPR, (d) DRP of the images.

Table-3: CNR, PCR and COC of reconstructed images of the object near electrode 5 .

\begin{tabular}{|l|l|l|l|}
\hline Regularization & CNR & PCR & COC \\
\hline LMR & 2.92 & 30.32 & 1.98 \\
\hline PEPR & 3.46 & 44.74 & 2.40 \\
\hline
\end{tabular}

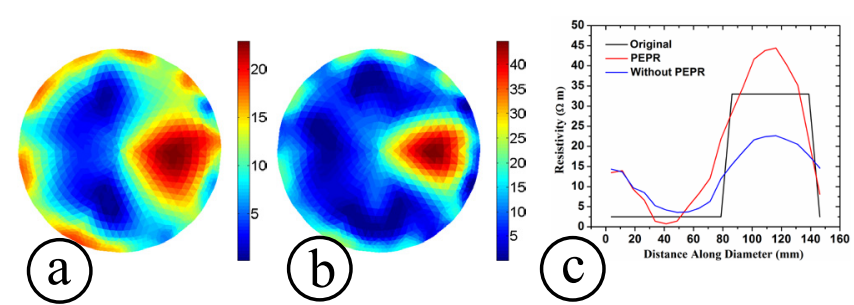

Fig.-5: Resistivity images with noisy $(25 \%)$ boundary data (object near electrode 1): (a) with LMR, (b) with PEPR, (c) DRP of the reconstructed image

Table-4: CNR, PCR and COC of reconstructed images for noisy data (object near electrode 1)

\begin{tabular}{|l|l|l|l|}
\hline Regularization & CNR & PCR & COC \\
\hline LMR & 1.81 & 27.29 & 1.85 \\
\hline PEPR & 3.17 & 63.79 & 2.90 \\
\hline
\end{tabular}

Resistivity images reconstructed from the noisy boundary data of the phantom with object near electrode 3 show that the CNR of the image reconstructed with LMR technique (Fig.-6a) is 1.60. On the other hand, CNR with PEPR (Fig.-6b) is 1.89 (Table-5). The PCR of the resistivity image with LMR technique is 23.61 whereas the PCR with PEPR is 35.68 (Table-5). It is also noticed that the COC of the reconstructed image with LMR is 1.73 but with PEPR it is 2.12 (Table-5). It is observed that the DRP of the resistivity image with PEPR (Fig.-6c) is more similar to the DRP of the original object.

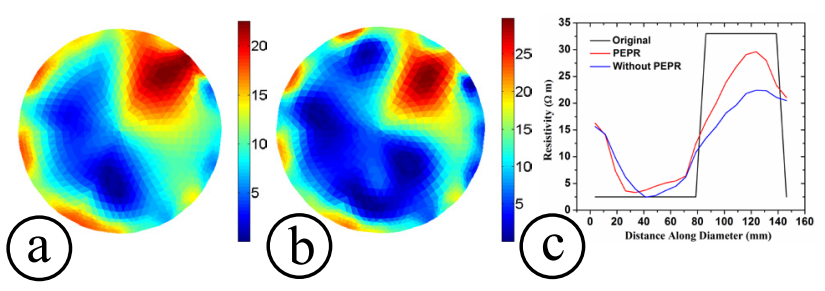

Fig.-6: Resistivity images with noisy (25\%) boundary data (object near electrode 3): (a) with LMR, (b) with PEPR, (c) DRP of the reconstructed image

Table-5: CNR, PCR and COC of reconstructed images for noisy data (object near electrode 3 )

\begin{tabular}{|l|l|l|l|}
\hline Regularization & CNR & PCR & COC \\
\hline LMR & 1.60 & 23.61 & 1.73 \\
\hline PEPR & 1.89 & 35.68 & 2.12 \\
\hline
\end{tabular}

It is also observed that for the resistivity images reconstructed from the noisy data for the phantom with object near electrode 5 , the CNR of the image reconstructed with LMR technique (Fig.-7a) is 1.24. On the other hand, CNR with PEPR (Fig.-7b) is 1.48 (Table-6). The PCR of the resistivity image with LMR technique is 17.59 whereas the PCR with PEPR is 25.91 (Table-6). It is also noticed that the COC of the resistivity image with LMR is 1.54 but with PEPR, it is 1.78 (Table-6). Result shows the DRP of the resistivity image with PEPR (Fig.-7c) is more similar to the DRP of the original object.

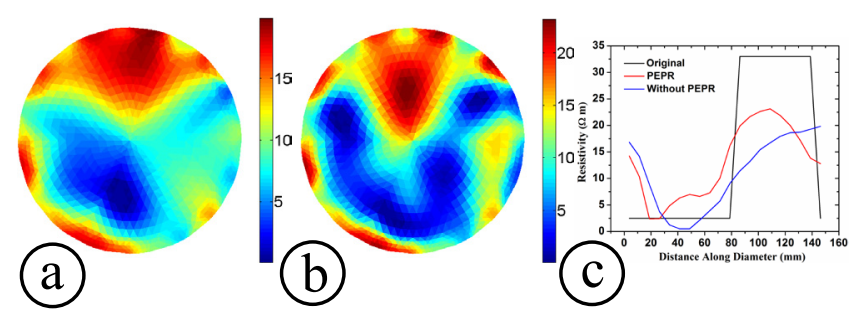

Fig.-7: Resistivity images with noisy $(25 \%)$ boundary data (object near electrode 5): (a) with LMR, (b) with PEPR, (c) DRP of the reconstructed image

Table-6: CNR, PCR and COC of reconstructed images for noisy data (object near electrode 5)

\begin{tabular}{|l|l|l|l|}
\hline Regularization & CNR & PCR & COC \\
\hline LMR & 1.24 & 17.59 & 1.54 \\
\hline PEPR & 1.48 & 25.91 & 1.78 \\
\hline
\end{tabular}




\section{Studies on PEPR and LMR with $\lambda=\Psi$}

In the above study, for the LMR method the $\lambda$ is taken as $10^{-1}$ in the first iteration and it is then modified as $\lambda / \mathrm{k}$ in the modified EIDORS, where $\mathrm{k}$ is the number of iterations. For further analysis the resistivity reconstruction is also conducted with a range of regularization parameters. Regularization effect, normalized projection error (error due to the voltage mismatch) and the normalized solution error norm are studied for different iterations. Result show that the resistivity image with PEPR $(\Psi=0.01)$ is found better compared to the image obtained for LMR with a $\lambda=$ 0.01 at first iteration and then it is decreased by a factor of $\sqrt{10}$ for the other iterations [25]. It is observed that CNR of the image reconstructed with LMR technique (Fig.-8a) is 2.95. On the other hand, CNR with PEPR (Fig.-8c) is 3.26 (Table-7). Due to the over-estimate of the inhomogeneity resistivity (Fig.-8b) the PCR of the resistivity image with LMR technique is very high compared to PEPR. For the similar reason COC is also found higher than the PEPR method. But $\mathrm{IR}_{\text {Max }}$ and $\mathrm{IR}_{\text {Mean }}$ are both suitable in PEPR method whereas, in LMR technique, they are very absurd compared to the original value (Table-7).

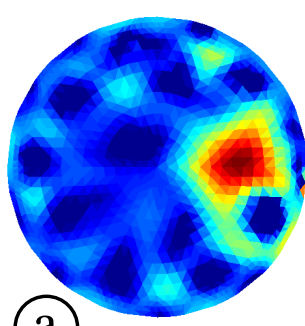

(a)

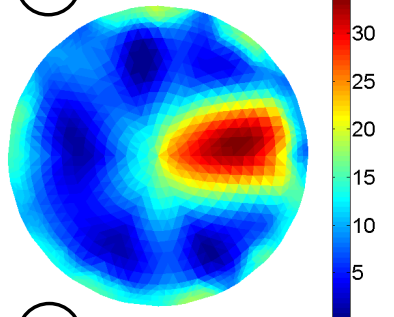

(c)

Fig.-8: Resistivity images obtained from boundary data with $10 \%$ noise (object near electrode 1): (a) image with LMR, (b) DRP of the reconstructed image shown in Fig.-1a, (c) image with PEPR, (d) DRP of the reconstructed image shown in Fig.-1c.

Table-7: CNR, PCR, COC, $\mathrm{IR}_{\mathrm{Max}}$ and $\mathrm{IR}_{\text {Mean }}$ of reconstructed images shown in Fig. -8 .

\begin{tabular}{|l|c|l|l|l|l|}
\hline Regularization & CNR & PCR & COC & IR $_{\text {Max }}$ & IR $_{\text {Mean }}$ \\
\hline LMR & 2.95 & 89.15 & 2.90 & 77.03 & 41.46 \\
\hline PEPR & 3.26 & 46.48 & 2.49 & 33.96 & 23.70 \\
\hline
\end{tabular}

Studies on PEPR and LMR in Different Iterations with $\lambda=\Psi$

Resistivity imaging with LMR $(\lambda=0.01)$ and PEPR $(\Psi=$ 0.01 ) techniques is studied for a number of iterations (result for first 12 iterations is presented). It is observed that resistivity images in LMR method (Fig.-9), the reconstruction diverges gradually after second iteration and become unstable with a continuously over-estimated resistivity (Fig.-10). On the other hand, the resistivity reconstruction rapidly converges in the PEPR method (Fig.-11) and gets stable after few iterations with a proper resistivity reconstruction (Fig.-12).

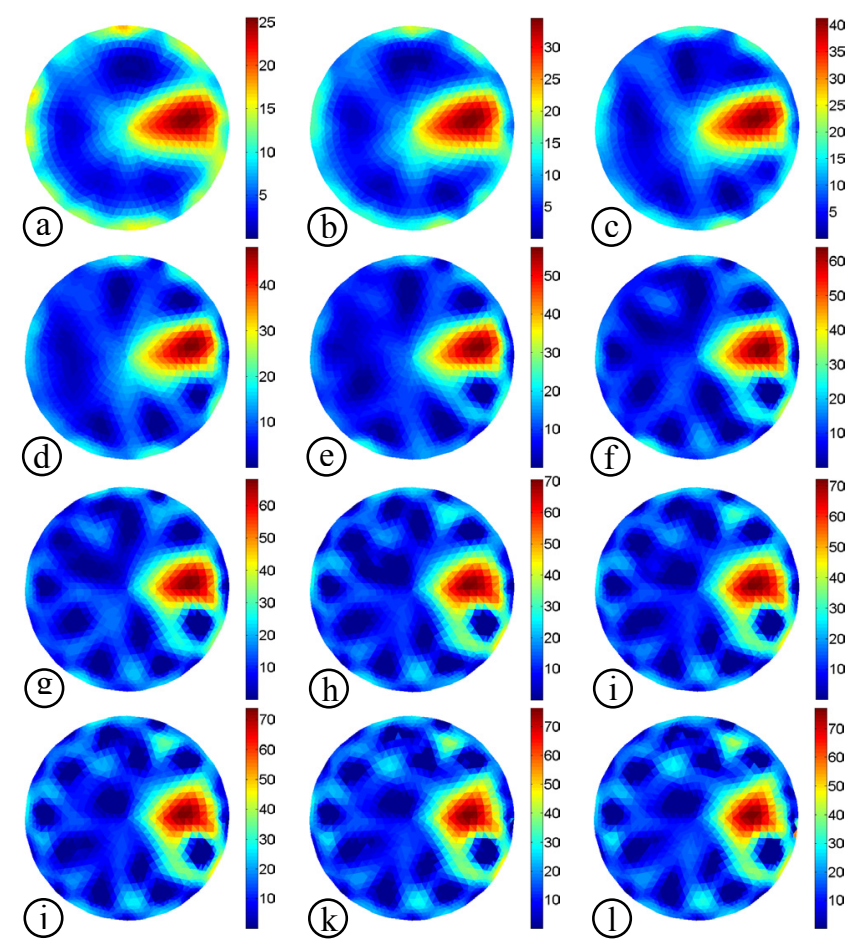

Fig.-9: Resistivity images obtained from noisy boundary data (Error added $=10 \%$ ) with LMR $(\lambda=0.01)$ method (object near electrode 1) for first twelve iterations: (a) to (1) images represents the reconstruction of 1 to 12 iterations respectively.
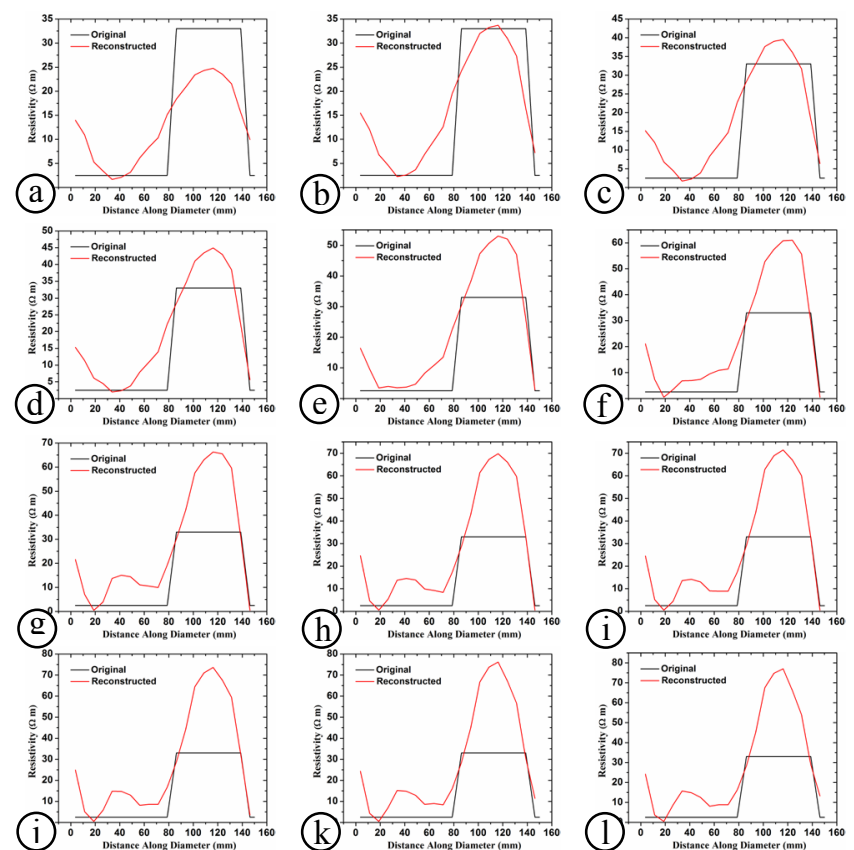

Fig.-10: DRP of the resistivity images shown in Fig.-9 (reconstruction with LMR): with noisy data (object near electrode 3): (a) to (1) images represents the DRP of the images shown in Fig.-9a to Fig.-91 respectively. 

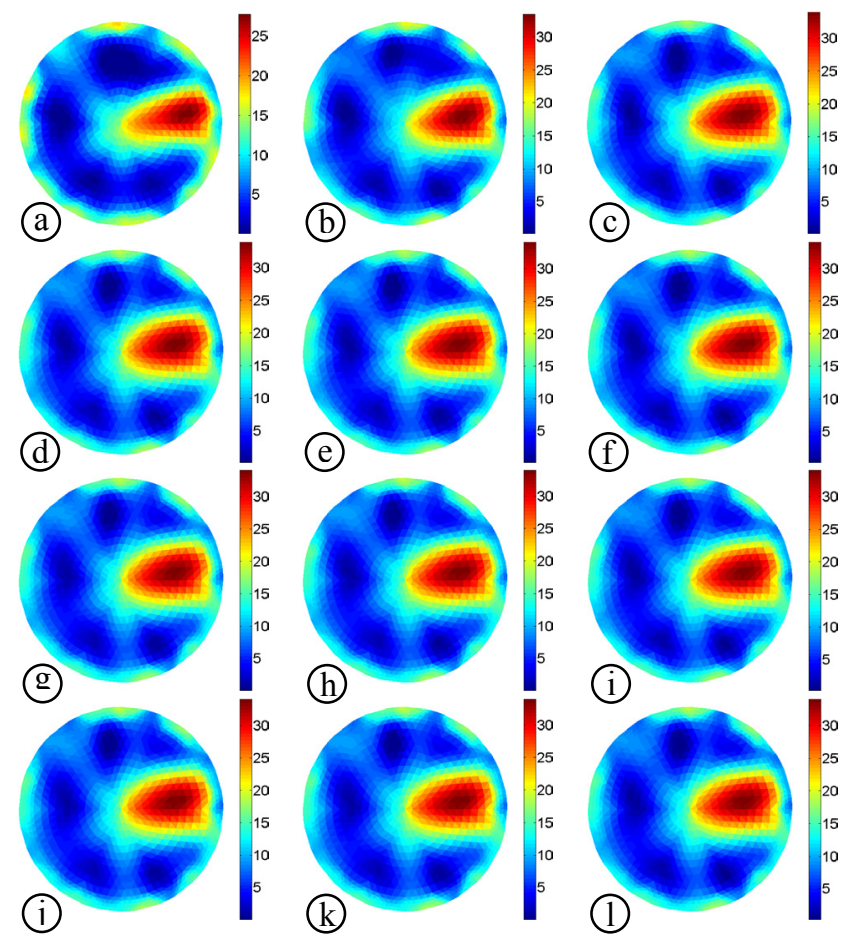

Fig.-11: Resistivity images obtained from noisy boundary data (Error added $=10 \%)$ with PEPR $(\Psi=0.01)$ method (object near electrode 1) for first twelve iterations: (a) to (1) images represents the reconstruction of 1 to 12 iterations respectively.
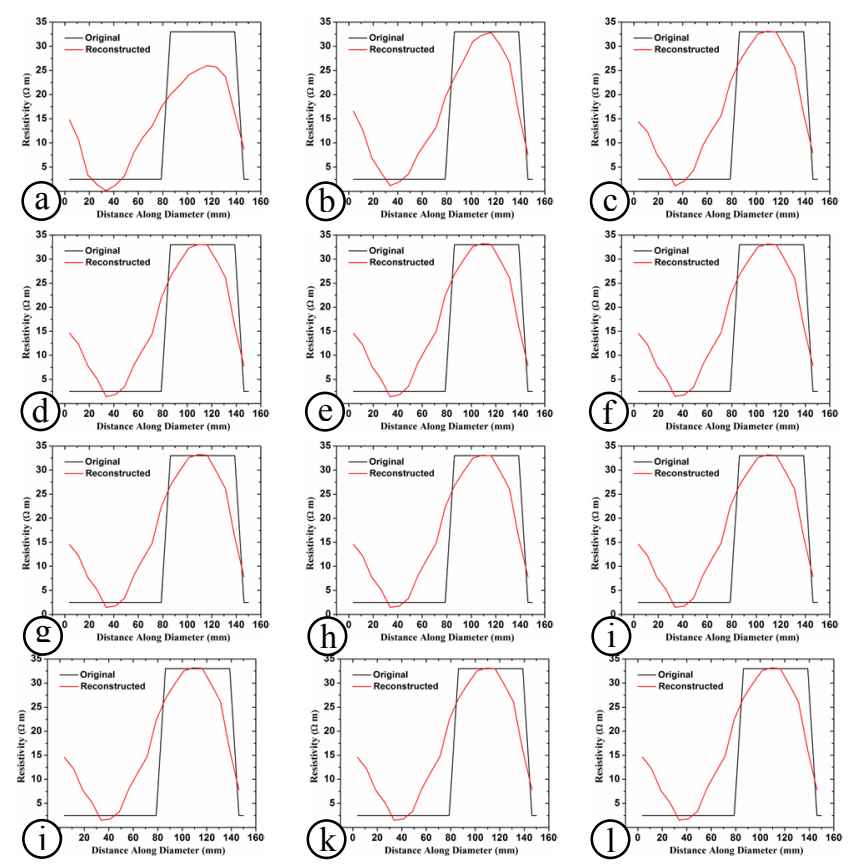

Fig.-12: DRP of the resistivity images shown in Fig.-11 (reconstruction with PEPR): with noisy data (object near electrode 1): (a) to (1) images represents the DRP of the images shown in Fig.-11a to Fig.-111 respectively.

\section{Studies on Projection Error and Solution Error Norm}

It is observed that the $\mathrm{IR}_{\mathrm{Max}}$ of the resistivity images with PEPR technique is more stable and closer to the original value, except for the first iteration (Fig.-13a). On the other hand, in $L M R, I_{\text {Max }}$ is larger than the original value (Fig.- 13a) except in first iteration $\left(\mathrm{IR}_{\mathrm{Max}}<\mathrm{IR}_{\text {Original }}\right)$ and the second iteration $\left(\mathrm{IR}_{\mathrm{Max}} \sim \mathrm{IR}_{\text {Original }}\right)$. Result show that, for PEPR, the standard deviation of the $\mathrm{IR}_{\mathrm{Max}}$ during first twelve iterations is 1.79 whereas it is 17.59 in LMR method (Fig.-13a).

Result show that $I_{\text {Mean }}$ of the resistivity images with the PEPR method is more stable whereas it is comparatively largely variable in the LMR method (Fig.-13b). Result show that, for PEPR, the standard deviation of the $\mathrm{IR}_{\text {Mean }}$ during the first twelve iterations is $1.79 \Omega \mathrm{m}$ whereas it is $8.21 \Omega \mathrm{m}$ in LMR method (Fig.-13b).

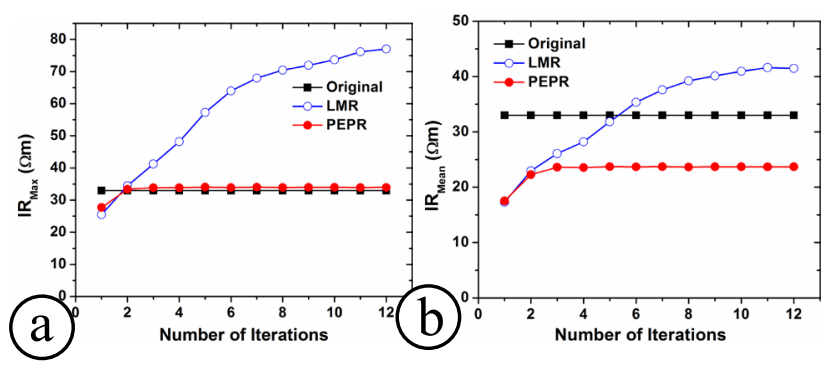

Fig.-13: Reconstruction parameters and reconstruction errors for the resistivity images obtained from noisy boundary data (Error added $=10 \%$ ) with LMR $(\lambda=0.01)$ and PEPR $(\Psi=0.01)$ methods (object near electrode 1): (a) maximum values of the reconstructed inhomogeneity resistivity ( $\left(R_{\text {Max }}\right)$, (b) mean of reconstructed inhomogeneity resistivity ( $\mathbb{R}_{\text {Mean }}$ ).

It is observed that the projection error $\left(E_{V}\right)$ in $L M R$ method is comparatively large and it is gradually increasing after third iteration (Fig.-14a). On the other hand, in PEPR, $\mathrm{E}_{\mathrm{V}}$ is comparatively low and almost constant after second iteration (Fig.-114a). Result show that, in the PEPR method, the normalized solution error norm $\left(\mathrm{E}_{\rho}\right)$ is less and varies from 0.74 to 0.77 (Fig.-14b). But, in the LMR method, $E_{\rho}$ is comparatively large and varies from 0.73 to 1.31 (Fig.-14d).
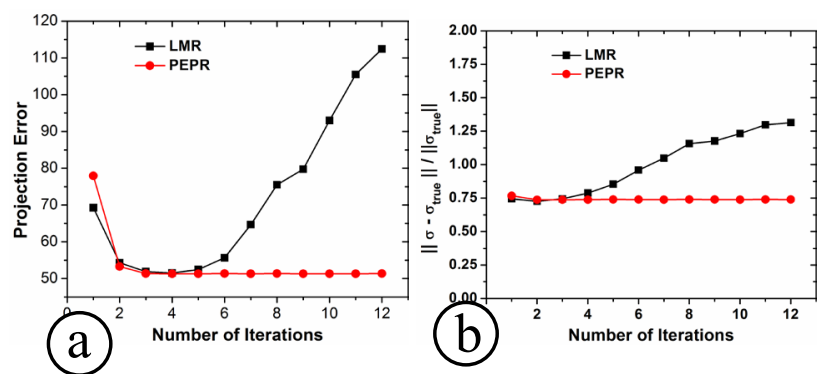

Fig.-14: Reconstruction parameters and reconstruction errors for the resistivity images obtained from noisy boundary data (Error added $=10 \%)$ with $\operatorname{LMR}(\lambda=0.01)$ and PEPR $(\Psi=0.01)$ methods (object near electrode 1): (a) projection error $\left(E_{V}\right),(b)$ normalized solution error norm $\left(\mathrm{E}_{\rho}\right)$.

\section{Studies on Different Values of $\lambda$ and $\Psi$}

The projection errors $\left(\mathrm{E}_{\mathrm{V}}\right)$ are calculated for different values of $\lambda$ in LMR (Fig.-15a) and different values of $\Psi$ in the PEPR method (Fig.-15b). It is observed that, for the LMR method, the $\mathrm{E}_{\mathrm{V}}$ becomes minimum at the third 
iteration for $\lambda=1.0$ and 0.1 whereas for $\lambda=0.01,0.001$ and 0.0001 , the $E_{V}$ becomes minimum at the fourth iteration (Fig.-15a). On the other hand, result show that (Fig.-15b), for the PEPR method, the $\mathrm{E}_{\mathrm{V}}$ becomes minimum at the third iteration for $\Psi=1.0$ and $0.1,0.01$ and 0.001 whereas for $\Psi$ $=0.0001$, the $\mathrm{E}_{\mathrm{V}}$ becomes minimum at the fourth iteration (Fig.-15a). Hence it is observed that the optimum reconstruction (i.e. the $E_{V}$ is minimum) occurs in PEPR with $\Psi=0.01$ where as the optimum reconstruction occurs in LMR with $\lambda=0.1$ (Table-8). It is also observed that the projection errors are comparatively less (Fig.-15b) in the PEPR method for all the values of $\Psi$. Result show that the projection errors are comparatively more stable in PEPR and they also become almost constant (Fig.-15b) after $3^{\text {rd }}$ iteration except for very low $\Psi(\Psi=0.0001)$.

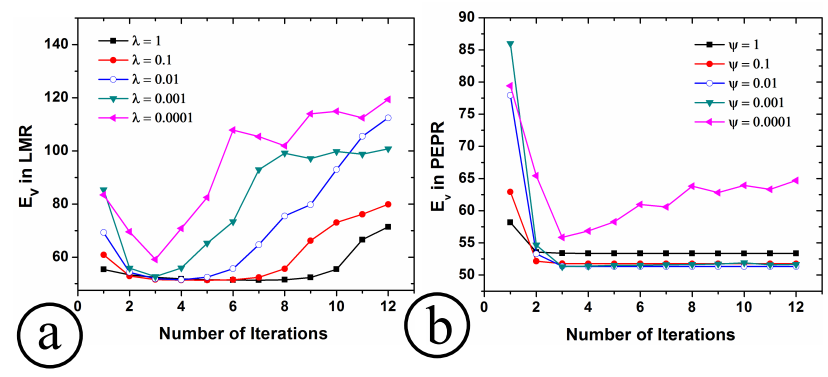

Fig.-15: Projection errors $\left(E_{V}\right)$ calculated in the resistivity reconstruction at different iterations (a) with $\operatorname{LMR}(\lambda=1$ to 0.0001$)$, (b) with PEPR ( $\Psi=1$ to 0.0001$)$.

Table-8: Projection errors $\left(E_{V}\right)$ calculated for different values of $\lambda$ in LMR and $\Psi$ in PEPR.

\begin{tabular}{|l|c|c|}
\hline \multicolumn{1}{|c|}{$\boldsymbol{\lambda}$ or $\boldsymbol{\Psi}$ at $\mathbf{4}^{\text {th }}$ Iteration } & LMR & PEPR \\
\hline 1.0 & 51.8248 & 53.3685 \\
\hline 0.1 & 51.3844 & 51.7703 \\
\hline 0.01 & 51.5016 & 51.3234 \\
\hline 0.001 & 55.9473 & 51.3802 \\
\hline 0.0001 & 70.8394 & 56.8654 \\
\hline
\end{tabular}

\section{Discussion}

A Projection Error Propagation-based Regularization (PEPR) method is proposed and the reconstructed image quality is improved in Electrical Impedance Tomography (EIT). The projection error is calculated from the difference between the calculated and measured data in each iterations of the reconstruction process and then it is integrated with the response matrix and the reconstruction is carried out. The PEPR method is studied with the simulated boundary data obtained for different inhomogeneity geometry. Studying the resistivity reconstruction from simulated data it is observed that the Projection Error Propagation-based Regularization (PEPR) method improved the quality of the reconstructed images in Electrical Impedance Tomography (EIT). CNR, PCR, COC, $I_{\text {Max }} I_{\text {Mean }}, E_{V}$ and $E_{\rho}$ all are improved in PEPR technique. Especially, the PEPR method improved the image quality for noisy boundary data. PEPR technique is also studied with the simulated boundary data mixed with random noise for different percentage. It is observed that the PEPR method gives better reconstruction at all the noise levels added to the boundary data. An iteration study shows that in LMR method, the reconstruction diverges gradually as the iteration goes on and become unstable with a continuously over estimated resistivity. On the other hand, the resistivity reconstruction rapidly converges in the PEPR method and gets stable after few iterations with a proper resistivity reconstruction. All the results demonstrate that the PEPR technique improves image reconstruction precision in EIDORS and hence it can be successfully implemented to increase the reconstruction accuracy in EIT.

\section{Conclusions}

PEPR technique is successfully implemented to regularize the solution domain in resistivity reconstruction in EIT. the PEPR method improves the image quality by increasing the CNR, PCR and COC for resistivity reconstruction in EIDORS. The simulation study proves that the PEPR technique improves the resistivity image quality with a better contrast than the traditional regularization for all inhomogeneity positions. Especially with noisy boundary data, the PEPR method provides improved reconstruction with high image contrast. Normalized projection error $\left(E_{V}\right)$ and the normalized solution error norm $\left(E_{\rho}\right)$ are found to be less in PEPR technique. It is observed that the resistivity reconstruction rapidly converges in the PEPR method and gets stable after few iterations with a proper resistivity reconstruction. On the other hand, in the LMR method, the reconstruction diverges gradually as the iteration goes on and become unstable with a continuously over-estimated resistivity. Hence it is observed that the PEPR is successfully implemented in EIDORS for better reconstruction in EIT.

\section{References}

1. Webster J. G. Electrical impedance tomography. Adam Hilger Series of Biomedical Engineering, Adam Hilger, New York, USA 1990.

2. Denyer C. W. L. Electronics for Real-Time and ThreeDimensional Electrical Impedance Tomographs, PhD Thesis, Oxford Brookes University, January 1996.

3. Metherall P. Three Dimensional Electrical Impedance Tomography of the Human Thorax, PhD Thesis, University of Sheffield. Jan'1998.

4. Huang C. N., Yu F. M. and Chung H. Y. The Scanning Data Collection Strategy for Enhancing the Quality of Electrical Impedance Tomography. IEEE Trans. Instrument. Meas. 2008;57(6):1193-1198. doi:10.1109/TIM.2007.915149 
5. Bushberg J. T., Seibert J. A., Leidholdt Jr. E. M., Boone J. M. The Essential Physics of Medical Imaging, 2nd Edition, Lippincott Williams \& Wilkins, ISBN-10: 0683301187. 2001.

6. $\quad$ Li Y., Rao L., He R., Xu G., Wu Q., Yan W., Dong G. and Yang Q. A Novel Combination Method of Electrical Impedance Tomography Inverse Problem for Brain Imaging. IEEE Trans. Magnetics. 2005;41(5):1848-1851. doi:10.1109/TMAG.2005.846506

7. Brown B. H. Medical impedance tomography and process impedance tomography: a brief review. Measurement Science \& Technology. 2001;12:991-996. doi:10.1088/0957-0233/12/8/301

8. Linderholm P., Marescot L., Loke M. H. and Renaud P. Cell Culture Imaging Using Microimpedance Tomography. IEEE Trans. on Biomed. Eng. 2008;55(1):138-146. doi:10.1109/TBME.2007.910649

9. Martinsen Ø. G., Kalvøy H., Grimnes S., Nordbotten B., Hol P. K., Fosse E., Myklebust H. and Becker L. B. Invasive Electrical Impedance Tomography for Blood Vessel Detection. The Open Biomed. Eng. J. 2010;4:135-137. doi: $10.2174 / 1874120701004010135$

10. Borsic A., Halter R., Wan Y., Hartov A. and Paulsen K. D. Electrical impedance tomography reconstruction for threedimensional imaging of the prostate. Physiol. Meas. 2010;31:S1-S16. doi:10.1088/0967-3334/31/8/S01

11. Bagshaw A. P., Liston A. D., Bayford R. H., Tizzard A., Gibson A. P., Tidswell A. T., Sparkes M. K., Dehghani H., Binnie C. D. and Holder D. S. Electrical impedance tomography of human brain function using reconstruction algorithms based on the finite element method. NeuroImage 2003;20:752-764. doi:10.1016/S1053-8119(03)00301-X

12. Murphy D., Burton P., Coombs R., Tarassenko L. and Rolfe P. Impedance Imaging in the Newborn. Clin. Phys. Physiol. Meas. 1987;8(Suppl. A):131-40. doi:10.1088/0143-0815/8/4A/017

13. Tyna H. A. and Iles S. E. Technology review: The use of electrical impedance scanning in the detection of breast cancer. Breast Cancer Research. 2004;6(2):69-74.

14. Moura F. S., Aya J. C. C., Fleury A. T., Amato M. B. P., and Lima R. G. Dynamic Imaging in Electrical Impedance Tomography of the Human Chest With Online Transition Matrix Identification. IEEE Trans. Biomed. Eng. 2010;57(2):422-431. doi:10.1109/TBME.2009.2032529

15. Ferraioli F., Formisano A., and Martone R. Effective Exploitation of Prior Information in Electrical Impedance Tomography for Thermal Monitoring of Hyperthermia Treatments. IEEE Trans. Magnetics. 2009;45(3):1554-1557. doi:10.1109/TMAG.2009.2012740

16. McArdle F. J., Suggett A. J., Brown B. H., and Barber D. C. An assessment of dynamic images by applied potential tomography for monitoring pulmonary perfusion. Clin. Phys. Physiol. Meas. 1988;9(Suppl. A):87-91. doi:10.1088/0143-0815/9/4A/015

17. Hoetink A. E., Faes T. J. C., Marcus J. T., Kerkkamp H. J. J. and Heethaar R. M. Imaging of Thoracic Blood Volume Changes During the Heart Cycle With Electrical Impedance
Using a Linear Spot-Electrode Array. IEEE Tran. on Med. Imaging. 2002;21(6):653-661. doi:10.1109/TMI.2002.800582

18. Ferrer A. R. Z., Castro G. M., Gaona G. A., Aguillon M.A., Rosell F. P. J. and Carrera B. J. Electrical Impedance Tomography: An Electronic Design, with Adaptive Voltage Measurements and A Phantom Circuit for Research in The Epilepsy Field, Proceedings - 19th Internl Conf. IEEE/EMBS Oct. 30 - Nov. 2, 1997, pp 867-868, USA.

19. Henderson R. P., Webster J. G. An impedance camera for spatially specific measurements of the thorax. IEEE Transactions on Biomedical Engineering. 1978;Bme25(3):250-254. doi:10.1109/TBME.1978.326329

20. Hou W. D., and Mo Y. L. Increasing image resolution in electrical impedance tomography. Electronics Letters. 2002;38:701-702. doi:10.1049/el:20020477

21. Lionheart W. R. B. EIT reconstruction algorithms: pitfalls, Review Article, challenges. Physiol. Meas. 2004;25:125-142. doi:10.1088/0967-3334/25/1/021

22. Wei. D. H. and Yu-Long M. New Regularization Method in Electrical Impedance Tomography. Journal of Shanghai University (English Edition) . 2002;6(3):211-215. doi:10.1007/s11741-002-0036-X

23. Vauhkonen M., Vadasz D., Karjalainen P. A., Somersalo E., and Kaipio J. P. Tikhonov Regularization and Prior Information in Electrical Impedance Tomography. IEEE Transactions on Medical Imaging. 1998;17(2):285-293. doi:10.1109/42.700740

24. B. W. Pogue, C. Willscher, T. O. McBride, U. L. Osterberg, and K. D. Paulsen. Contrast-detail analysis for detection and characterization with near-infrared diffuse tomography. Med. Phys. 2000;27:2693-2700. doi:10.1118/1.1323984

25. Niu H., Guo P., Ji L., Zhao Q. and Jiang T. Improving image quality of diffuse optical tomography with a projection-errorbased adaptive regularization method. Optics Express. 2008;16(17):12423. doi:10.1364/OE.16.012423

26. Polydorides N. and Lionheart W. R. B. A Matlab toolkit for three-dimensional electrical impedance tomography: a contribution to the Electrical Impedance and Diffuse Optical Reconstruction Software project. Meas. Sci. Technol. 2002;13:1871-1883. doi:10.1088/0957-0233/13/12/310

27. Vauhkonen M., Lionheart W. R. B., L. M. Heikkinen, P. J. Vauhkonen, J. P. Kaipio. A Matlab package for the EIDORS project to reconstruct two dimensional EIT images. Physiol. Meas. 2001;22:107-111. doi:10.1088/0967-3334/22/1/314

28. Bera T. K. and Nagaraju J. A Stainless Steel Electrode Phantom to Study the Forward Problem of Electrical Impedance Tomography (EIT). Sensors \& Transducers Journal. 2009;104(5):33-40.

29. Bera T. K. and Nagaraju J. A Reconfigurable Practical Phantom for Studying the 2 D Electrical Impedance Tomography (EIT) Using a FEM Based Forward Solver, 10th International Conference on Biomedical Applications of Electrical Impedance Tomography (EIT 2009), School of Mathematics, The University of Manchester, UK, 16th-19th June 2009. 
30. Bera T. K. and Nagaraju J. A Study of Practical Biological Phantoms with Simple Instrumentation for Electrical Impedance Tomography (EIT), Proceedings of IEEE International Instrumentation and Measurement Technology Conference (I2MTC2009), Singapore, 5th - 7th May 2009, pp 511-516.

31. Bera T. K. and Nagaraju J. Studying the Boundary Data Profile of A Practical Phantom for Medical Electrical Impedance Tomography with Different Electrode Geometries, Proceedings of The World Congress on Medical Physics and Biomedical Engineering-2009 Sept 7-12, 2009, Munich, Germany, IFMBE Proceedings 25/II, pp. 925-929.

32. Malmivuo J. and Plonsey R. Bioelectromagnetism: principles and applications of bioelectric and biomagnetic fields, Chapter-26, Sec.-26.2.1, New York, Oxford University Press, 1995.

33. Bera T. K. and Nagaraju J. A Simple Instrumentation Calibration Technique for Electrical Impedance Tomography (EIT) Using A 16 Electrode Phantom, Proceedings of The Fifth Annual IEEE Conference on Automation Science and Engineering (IEEE CASE 2009), Bangalore, August 22 to 25, pp. 347-352.

34. Brown B. H., Barber D. C., A. D. Seagar. Applied potential tomography: possible clinical applications. Clin. Phys. Physiol. Meas. 1985;6:109-121. doi:10.1088/0143-0815/6/2/002

35. Graham B. M. Enhancements in Electrical Impedance Tomography (EIT) Image Reconstruction for 3D Lung Imaging, PhD thesis, University of Ottawa, April 2007.

36. Yorkey T. J. Comparing reconstruction methods for electrical impedance tomography, $\mathrm{PhD}$ thesis, University of. Wisconsin at Madison, Madison, WI 53706, 1986.

37. Reddy J. N. An Introduction to the Finite Element Method, 3rd Ed., 2nd Reprint, TATA McGraw-Hill Pub. Co. Ltd, 2006.

38. Biswas S. K., Rajan K., Vasu R. M. Interior photon absorption based adaptive regularization improves diffuse optical tomography, Proc. SPIE, Volume 7546, 754611 (2010). doi:10.1117/12.853421
39. Grootveld C. J. Measuring and Modeling of Concentrated Settling Suspensions Using Electrical Impedance Tomography, PhD Thesis, Delft University of Technology, The Netherlands, 1996.

40. Arridge S. R. Optical tomography in medical imaging, Topical Review. Inverse Problems. 1999;15:R41-R93. doi:10.1088/0266-5611/15/2/022

41. Soleimani M., Yalavarthy P. K. and Dehghani H. Helmholtztype regularization method for permittivity reconstruction using experimental phantom data of electrical capacitance tomography. IEEE Trans. Instrum. Meas. 2010;59(1):78-83. doi:10.1109/TIM.2009.2021645

42. M. Soleimani and W. R. B. Lionheart. Nonlinear image reconstruction in electrical capacitance tomography using experimental data. Meas. Sci. Technol., 2005;16(10):19871996. doi:10.1088/0957-0233/16/10/014

43. Chan T. F. and Tai X. C. Level set and total variation regularization for elliptic inverse problems with discontinuous coefficients. J. Comput. Phys. 2004;193(1):40 66. doi:10.1016/j.jcp.2003.08.003

44. Bera T. K. and Nagaraju J. Resistivity Imaging of A Reconfigurable Phantom With Circular Inhomogeneities in 2D-Electrical Impedance Tomography. Measurement. 2011;44(3):518-526. doi:10.1016/j.measurement.2010.11.015

45. Song X., Pogue B. W., Jiang S., Doyley M. M., Dehghani H., Tosteson T. D., and Paulsen K. D. Automated region detection based on the contrast-to-noise ratio in near-infrared tomography. Appl. Opt. 2004;43:1053-1062. doi:10.1364/AO.43.001053

46. Kanmani B. and Vasu R. M. Diffuse optical tomography using intensity measurements and the a priori acquired regions of interest: theory and simulations. Phys. Med. Biol. 2005;50:247-264. doi:10.1088/0031-9155/50/2/005

47. Reyes M., Malandain G., Koulibaly P. M., GonzálezBallester M. A. and Darcourt J. Model-based respiratory motion compensation for emission tomography image reconstruction. Phys. Med. Biol. 2007;52:3579-3600. doi:10.1088/0031-9155/52/12/016 